\title{
Study of an HHG-Seeded Free-Electron Laser for the LBNL Next Generation Light Source
}

\author{
Neil Thompson, ASTeC, CCLRC, Daresbury Laboratory
}

\begin{abstract}
DISCLAIMER
This document was prepared as an account of work sponsored by the United States Government. While this document is believed to contain correct information, neither the United States Government nor any agency thereof, nor The Regents of the University of California, nor any of their employees, makes any warranty, express or implied, or assumes any legal responsibility for the accuracy, completeness, or usefulness of any information, apparatus, product. or process disclosed, or represents that its use would not infringe privately owned rights. Reference herein to any specific commercial product, process, or service by its trade name, trademark, manufacturer, or otherwise, does not necessarily constitute or imply its endorsement, recommendation, or favoring by the United States Government or any agency thereof, or The Regents of the University of California. The views and opinions of authors expressed herein do not necessarily state or reflect those of the United States Government or any agency thereof or The Regents of the University of California.
\end{abstract}

This work was supported by the Director, Office of Science, Office of Fusion Energy Sciences, of the U.S. Department of Energy under Contract No. DE-AC02-05CHI1231. 
| 


\title{
Study of an HHG-Seeded Free-Electron Laser for the LBNL Next Generation Light Source
}

\author{
Neil Thompson, ASTeC, CCLRC Daresbury Laboratory
}

October 20, 2010

\section{Contents}

1 Introduction 1

2 Parameter Optimisation 2

2.1 Radiator Undulator Period ...................... 2

2.2 Modulator Undulator Period and Seed Tuning Range . . . . . . . . . . . 5

2.3 Electron Beam Focussing $\ldots \ldots \ldots \ldots \ldots \ldots$

2.4 Optimisation via Steady-State Simulations . . . . . . . . . . . . 6

3 Time Dependent Simulations $\quad 7$

3.1 Single Harmonic Conversion . . . . . . . . . . . . . . . . . . . 9 9

3.2 Two Harmonic Conversions $\ldots \ldots \ldots$

3.3 Reduced Seed Power . . . . . . . . . . . . . . . . . . . . . 11

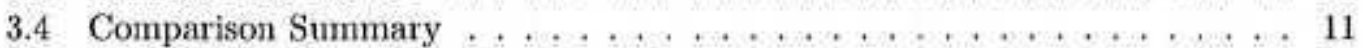

4 Harmonic Afterburners 13

4.1 Simulations ............................. 13

\section{Introduction}

The Next Generation Light Source (NGLS) is a high repetition rate free-electron laser facility proposed by Lawrence Berkeley National Laboratory (LBNL). The proposed facility will provide multiple FEL lines with varying spectral characteristics to satisfy a broad soft X-ray physics programme. At this stage of the project a number of FEL technologies and concepts are being investigated for possible implementation on the facility. In this report we consider a free-electron laser seeded by a Higher Harmonic Generation (HHG) source in which a high power (and consequently relatively low repetition rate) laser pulse is injected into a chamber of inert gas. Through a process of ionisation and recombination coherent higher harmonics of the laser are emitted from the gas and can be injected into an FEL system as a seed field. Further harnonic upeonversion can be done within the FEL system to enable temporally coherent FEL output at wavelengths much shorter than, and pulse energies orders of magnitude higher than, the HHG source emission.

The harmonic conversion within the FEL works in the following way. The seed field induces an energy modulation within the electron bunch at the start of the modulator. This energy modulation grows within the modulator due to the FEL interaction and starts to convert into a density modulation, or bunching, at the seed wavelength. However, this bunching also has components at higher harmonics which retain the longitudinal 
coherence of the initial seed. The beam passes through a magnetic chicane, which shears the longitudinal phase space to maximise the bunching at the required harmonic, then a further undulator which is tuned to this harmonic. If this second undulator is short it acts as a further modulator, and because the beam is pre-bunched at the modulator resonance there is a strong coherent burst of radiation which acts to modulate the electron beam energy in much the same way the input laser seed field acted in the first modulator. This second modulator is followed by a second bunching chicane and then a final long radiator tuned to a yet higher harmonic of the laser seed - the final output wavelength. Alternatively, the second undulator can be the radiator itself, in which case only one harmonic conversion from seed wavelength to final output is necessary.

We initially consider the case of a $400 \mathrm{~kW}$ peak power HHG seed source at wavelength $12 \mathrm{~nm}$ (currently considered the cutoff wavelength for sufficient seed power to dominate shot noise in the electron beam) which is converted in either one or two stages or harmonic conversion to FEL emission at $1 \mathrm{~nm}$. We then consider the implications of a factor of ten reduction in seed power to $40 \mathrm{~kW}$.

\section{Parameter Optimisation}

The primary requirement is to produce temporally coherent FEL pulses at an output wavelength of $1 \mathrm{~nm}$. Table 1 lists the parameters used for the electron beam and HHG seed source as well as given contraints on the undulators such as minimum gap, available space and required tuning range of the radiator undulators. All these values for the NGLS project are continually evolving with ongoing design work so the numbers stated here are a nominal set as of July 2010. The HHG seed source specification is taken from a design study done for the UK NLS Project [1] and represents the projected capability of a stateof-the-art source that could be built on the timescale of the NLS project build. The power given is that actually delivered to the FEL modulator undulator and accounts for losses due to transmission.

\subsection{Radiator Undulator Period}

An initial assessment of the required radiator undulator period was done using the design formulae of Ming Xie [3]. Figures 1 and 2 show, as a function of undulator gap $g$ on the $x$-axis and undulator period $\lambda_{w}$ on the $y$-axis, contours of resonant wavelength $\lambda_{t}$ (top left), 3D gain length $L_{g}$ (top right), $r m s$ undulator parameter $\bar{a}_{w}$ (bottom left) and saturation power $P_{\text {kat }}$ (bottom right). Figure 1 assumes an APPLE-II undulator in horizontal polarisation mode, and Figure 2 is the equivalent case for circularly polarised mode. The equations for undulator field us gap are taken from the FERMI@Elettra Conceptual Design Report [2]. The mean electron beam $\beta$-function used for each point on the $\left(g, \lambda_{w}\right)$ plane is the value which is found to minimise the gain length, constrained to vary over the range $4-50 \mathrm{~m}$.

The period finally chosen was $31 \mathrm{~mm}$. On the contour plots of resonant wavelength the red bar indicates the range of tuning, from $\lambda_{r}=4.25 \mathrm{~nm}(4.00 \mathrm{~mm})$ in horizontal (circular) polarisation at minimum gap of $5 \mathrm{~mm}$ to $\lambda_{r}=1.0 \mathrm{~nm}$ at gap of $17.7 \mathrm{~mm}(14.4 \mathrm{~mm})$. This red bar is then overlaid on the other three contour plots to show the corresponding variation in $L_{g}, \bar{a}_{w}$ and $P_{\text {sat }}$ for this range of gap tuning. It is clear from these figures that if the undulator period was increased to, for example, $\lambda_{w}=35 \mathrm{~mm}$, the tuning range would be extended to include longer wavelengths but the undulator parameter would fall well below $\bar{a}_{w}=0.7$ which is considered as a cut-off value for reasonable FEL coupling. 


\begin{tabular}{ll}
\hline \multicolumn{2}{c}{ ELECTRON BEAM } \\
\hline Electron beam energy $E$ & $2.4 \mathrm{GeV}$ \\
Normalised emittance $\varepsilon_{n}$ & $0.8 \mathrm{~mm}$-mrad \\
Energy Spread (rms) $\sigma_{E}$ & $100 \mathrm{keV}$ \\
Peak Current $I_{\text {peak }}$ & $1 \mathrm{kA}$ \\
\hline \multicolumn{2}{c}{ HHG SEED SOURCE } \\
\hline Peak Power $P_{\text {seed }}$ & $400 \mathrm{~kW}$ \\
Pulse duration (FWHM) $\tau_{\text {seed }}$ & $20 \mathrm{fs}$ \\
Minimum Wavelength $\lambda_{\text {min }}$ & $12 \mathrm{~nm}$ \\
Repetition rate $f$ & $1 \mathrm{kHz}$ \\
\hline \multicolumn{2}{c}{ UNDULATORS } \\
\hline Minimum gap $g_{\text {min }}$ & $5 \mathrm{~mm}$ \\
Radiator tuning range & $1-5 \mathrm{~mm}$ \\
Polarisation & Horizontal $\leftrightarrow$ Circular \\
Module length $L_{u}$ & $3 \mathrm{~m}$ \\
Intermodule gap $L_{\text {gap }}$ & $1 \mathrm{~m}$ \\
Length of undulator hall & $\simeq 60 \mathrm{~m}$ \\
\hline
\end{tabular}

Table 1: Summary of nominal parameters for electron beam and HHG seed source, as well as contraints on the undulator systems.
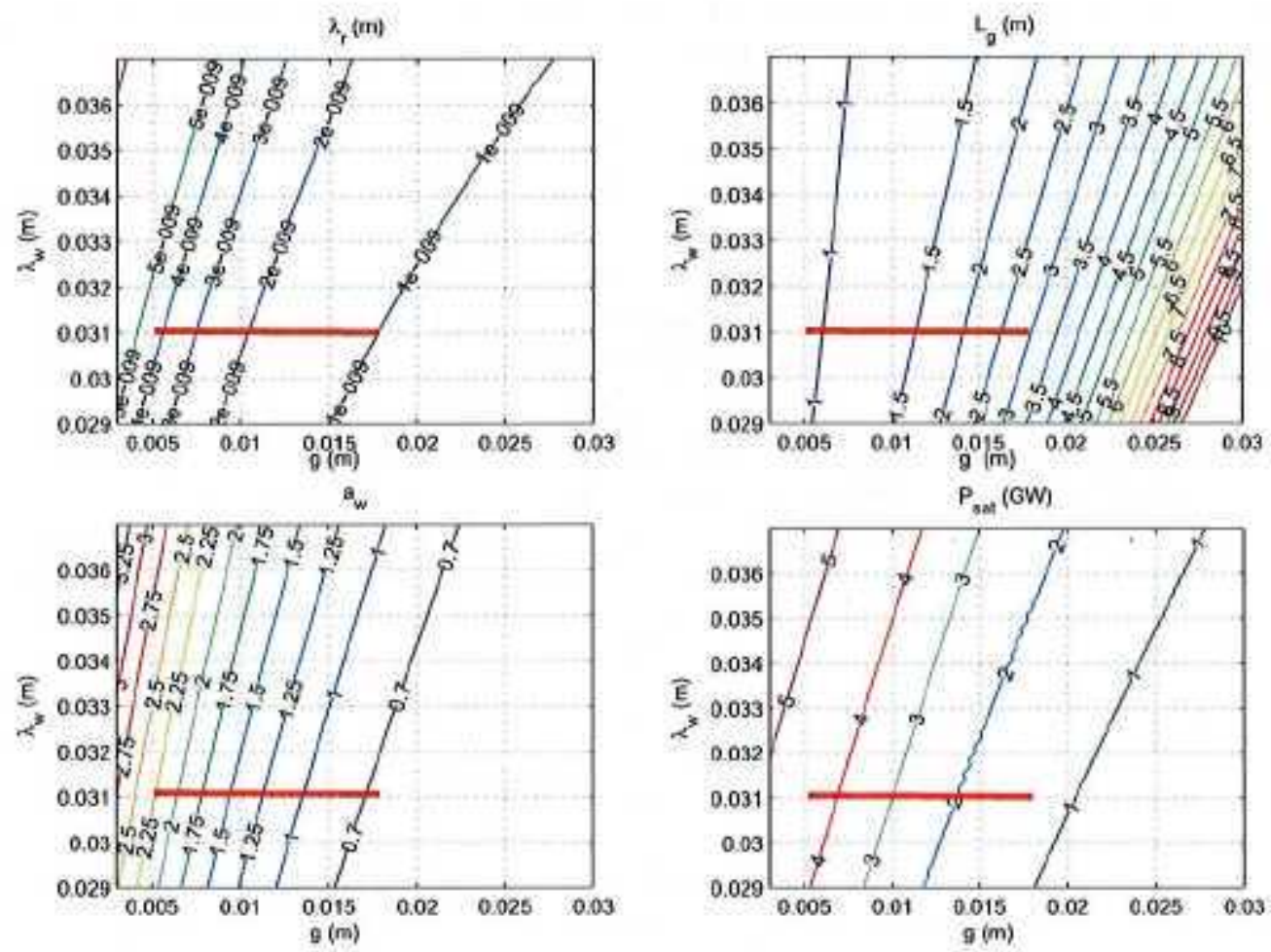

Figure 1: Choice of radiator undulator period - horizontal polarisation. 

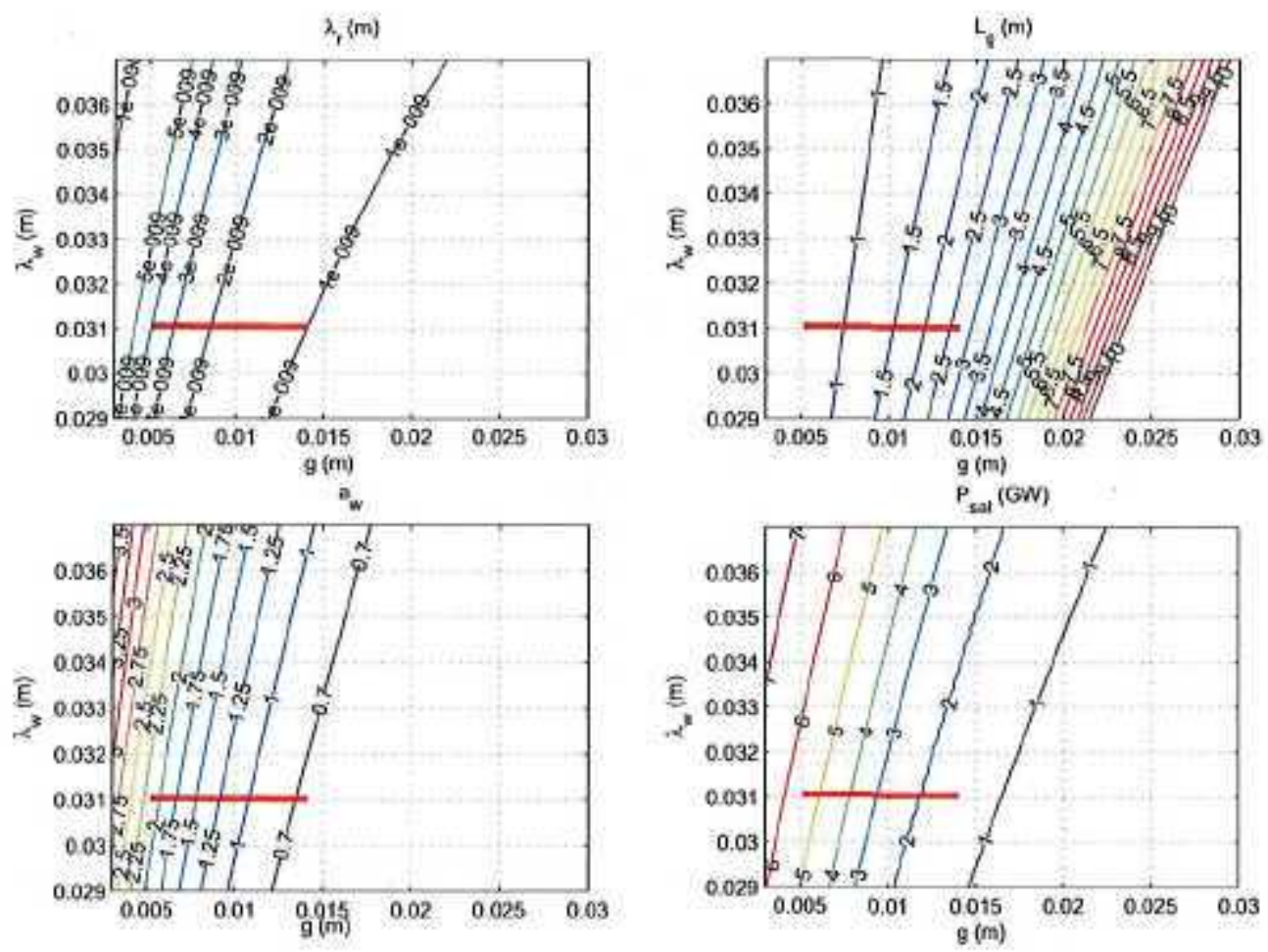

Figure 2: Choice of radiator undulator period - circular polarisation.

Therefore, in order to maintain sufficient FEL coupling at $1 \mathrm{~nm}$, with a given minimum gap of $5 \mathrm{~mm}$, the tuning range must be slightly constrained from the desired 1-5 $\mathrm{mm}$.

A crude estimate was made of the maximum allowed gain length for which the total length of undulator could be kept below the available length of $60 \mathrm{~m}$. The argument was that if the required power contrast ratio between the seeded section of the FEL pulse and the surrounding SASE pedestal is a factor of 100 , then assuming the same growth rate for seeded and unseeded sections of the beam the seeded power must be in effect $\ln (100)=5$ power gain lengths 'ahead' of the SASE background. SASE typically takes around 20 gain lengths to reach saturation, so the radiator undulator should need to be no more than 15 gain lengths long. With the further assumptions that 3 modulator undulators may be required, each one $3 \mathrm{~m}$ long with $1 \mathrm{~m}$ gaps between this leaves $48 \mathrm{~m}$ of the $60 \mathrm{~m}$ undulator hall for the radiator undulators. Again, assuming $3 \mathrm{~m}$ modules with $1 \mathrm{~m}$ gaps, or a packing efficiency of $75 \%$, the actual useful length of radiator undulator is $36 \mathrm{~m}$ so the gain length must be less than $36 \mathrm{~m} / 15=2.4 \mathrm{~m}$. From Figures 1 and 2 it is seen that at $1 \mathrm{~nm}$, for $\lambda_{w}=31 \mathrm{~mm}, L_{g}=2.9 \mathrm{~m}(2.7 \mathrm{~m})$ in horizontal (circular) mode, nearly satisfying the required estimated value. However, for $\lambda_{w}>31 \mathrm{~mm}$ gain length $L_{g}$ increases steadily, making it more difficult to accommodate the radiator undulators in the required space.

A fiurther test was done to confirm the choice of undulator period. Simulations were done, using Genesis 1.3 in steady-state (single slice) mode, to calculate the saturation length $L_{\text {aat }}$ (including intermodule gaps of $1 \mathrm{~m}$ ) and saturation power $P_{\text {sat }}$ as a function of the bunching factor $b_{0}$ at the entrance to the radiator. This was done with the undulator tuned to $1 \mathrm{~nm}$, for undulator periods of $\lambda_{w}=31 \mathrm{~mm}$ and $35 \mathrm{~mm}$. Two different input energy spreads of $\sigma_{E}=100 \mathrm{keV}$ and $300 \mathrm{keV}$ were used, with these values representing the nominal energy spread at the input to the system and an estimate of the energy spread that would be induced by the interaction in the modulator undulators. The results are 

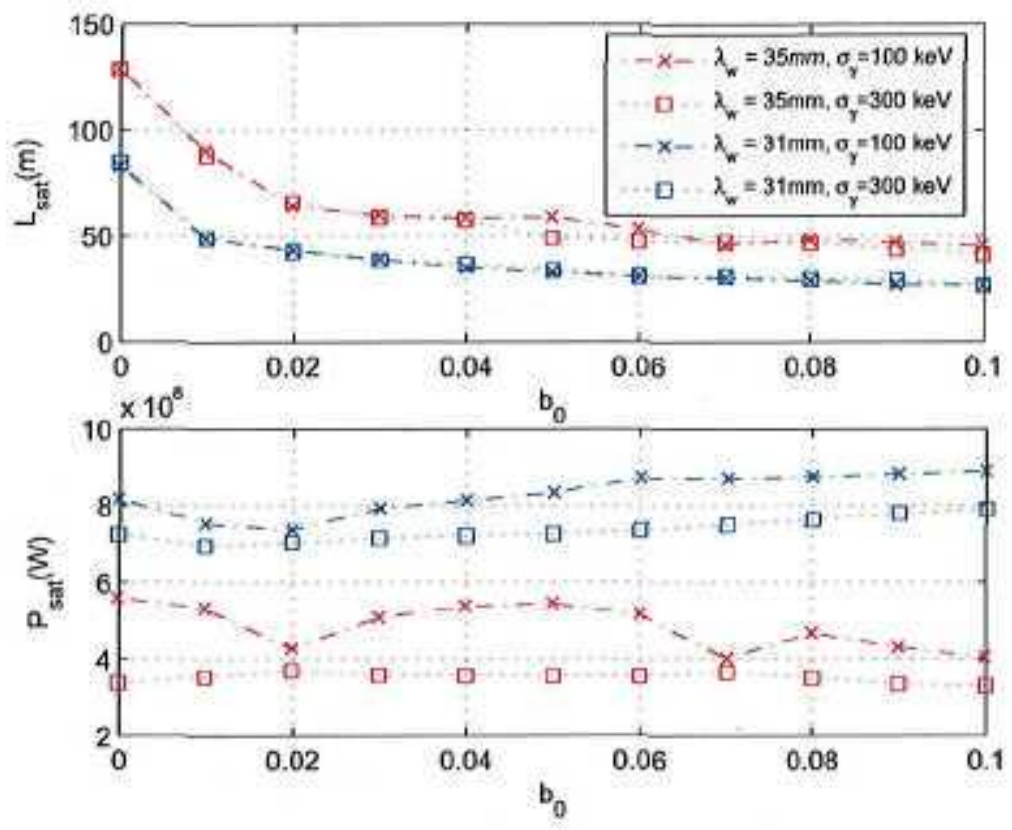

Figure 3: Saturation power and saturation length vs bunching factor $b_{0}$ at entrance to radiator.

shown in Figure 3. For $\lambda_{w}=35 \mathrm{~mm}$ the bunching factor needs to satisfy $b_{0}>0.07$ to keep $L_{\mathrm{sat}}<48 \mathrm{~m}$, whereas for $\lambda_{w}=31 \mathrm{~mm}$ the bunching required is much weaker at $b_{0}=0.01$. The latter value is a more realistic target-sufficient to ensure temporal coherence of the final FEL output yet allowing more contingency in the parameters of the modulator undulators and bunching chicanes. It is also demonstrated in Figure 3, in agreement with the Xie calculations of Figures 1 and 2, that the saturation power is significantly higher for the shorter period undulator.

To summarise, the chosen undulator period for the radiator undulators is $\lambda_{w}=31 \mathrm{~mm}$. This slightly restricts the tuning range from the preferred $1-5 \mathrm{~mm}$ but gives sufficient FEL coupling at $1 \mathrm{~nm}$ to allow saturation to be reached within the available length of the NGLS undulator hall.

\subsection{Modulator Undulator Period and Seed Tuning Range}

The tuning range of the $31 \mathrm{~mm}$ period radiator undulator is $1-4.25 \mathrm{~nm}$, as discussed in Section 2.1. The seed minimum wavelength is $12 \mathrm{~nm}$ so to reach $1.0 \mathrm{~nm}$ conversion to the $12 \mathrm{th}$ harmonic is required. This leaves a number of possible combinations of either one or two harmonic jumps to cover the full required tuning range. The design for the NLS HHG seed source provided continuous tunability from 24-12 $\mathrm{nm}$ so this range is adopted for the NGLS design. On this basis the tuning ranges of the modulator undulators are determined as follows:

- Modulator 1: Tunes over $25.5 \mathrm{~nm} \rightarrow 12.0 \mathrm{~nm}$. There is then a third harmonic jump to Modulator 2.

- Modulator 2: Tunes over $8.5 \mathrm{~nm} \rightarrow 4.0 \mathrm{~mm}$. There is then a $2 \mathrm{nd}, 3 \mathrm{rd}$ or 4 th harmonic jump to the radiator.

- Radiator: Thnes over $4.25 \mathrm{~nm} \rightarrow 1.0 \mathrm{~nm}$ 

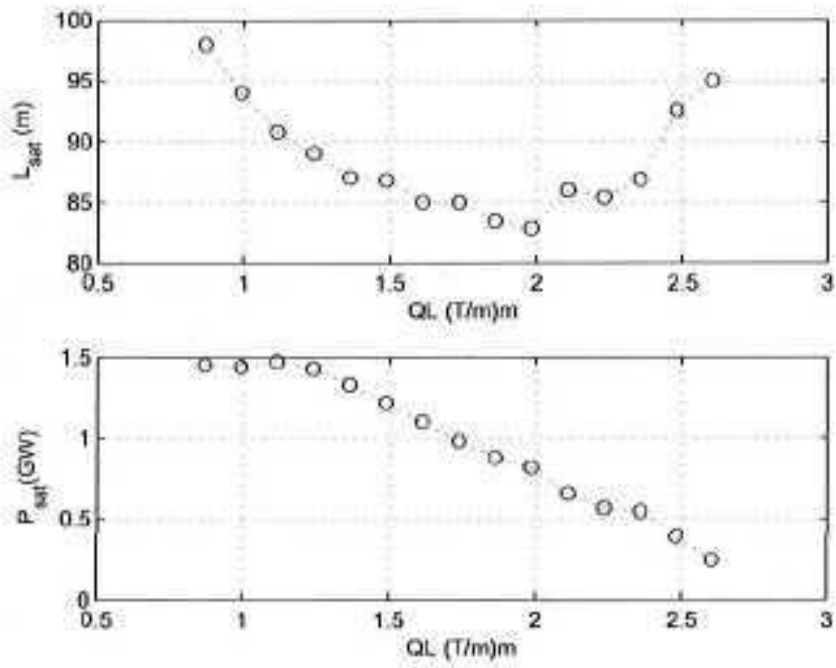

Figure 4: Saturation power and saturation length vs FODO focussing strength $Q L$.

To allow flexibility in changing the balance of undulator lengths at the different harmonic stages it is an advantage to use the same undulator period for both types of modulator. In this way any modulator undulator can be used as either a Modulator 1 or a Modulator 2. The period is therefore chosen to give full tunability from $25.5 \mathrm{~nm}$ to $4.0 \mathrm{~nm}$. For a planar PPM undulator a period of $\lambda_{w}=49 \mathrm{~mm}$ is appropriate, with minimum operating gap of $5 \mathrm{~mm}$. This gives a strong undulator parameter over the whole tuning range, varying from $\bar{a}_{w}=4.7$ at $25.5 \mathrm{~nm}$ to $\bar{a}_{w}=3.1$ at $12 \mathrm{~nm}$.

\subsection{Electron Beam Focussing}

A FODO focussing lattice is assumed. The dependence of SASE saturation length $L_{\text {sat }}$ and saturation power $P_{\mathrm{sat}}$ on the integrated quadrupole strength $Q L$, with $Q$ the field gradient in $\mathrm{T} / \mathrm{m}$ and $L$ the magnet length, is shown in Figure 4. The data is from steady state Genesis 1.3 simulations of the radiator undulator only. The working point is chosen. as that which minimises $L_{\text {sat, }}$ giving $Q L=1.98(\mathrm{~T} / \mathrm{m}) \mathrm{m}$.

\subsection{Optimisation via Steady-State Simulations}

The optimisation of the system was done using Genesis 1.3 in steady state mode. The strategy when determining the modulator lengths was to allow the energy modulation induced by the seed field within the modulator to become sufficiently large that it is possible to create strong bunching at the required harmonic number with the subsequent bunching chicane. Quantitatively this is expressed by the criterion

$$
\Delta \gamma>n \sigma_{\gamma}
$$

with $\Delta \gamma$ the full depth of the induced energy modulation, $n$ the required harmonic number and $\sigma_{\gamma}$ the natural rms energy spread in the beam. Simultaneously however, the energy modulation must not be sufficiently large that it inhibits exponential amplifieation in the radiator. Again a criterion can be applied that the total energy spread at the start of the radiator, found by combining the natural energy spread and induced energy modulation 


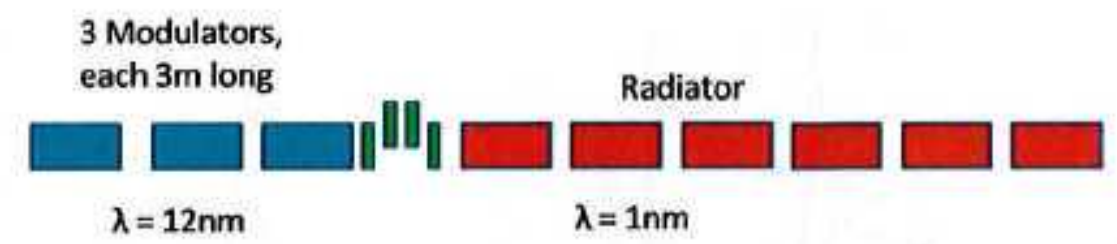

Figure 5: Single harmonic conversion scheme: schematic layout.

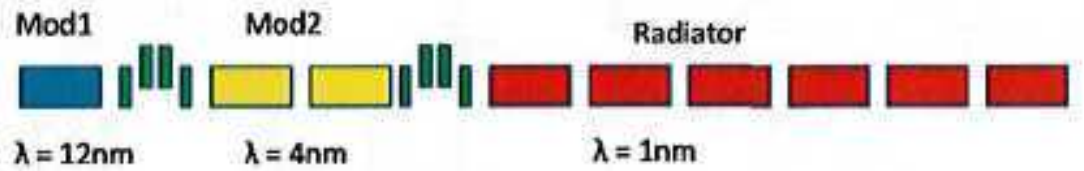

Figure 6: Double harmonic conversion scheme: schematic layout.

in quadrature, must satisfy

$$
\sigma_{\gamma, \text { total }}=\sqrt{\sigma_{\gamma}^{2}+\left(\frac{\Delta \gamma}{\sqrt{2}}\right)^{2}}<\rho \gamma
$$

with $\rho$ the usual FEL parameter. Combining (1) and (2) gives the range of acceptable energy modulation

$$
n \sigma_{\gamma}<\Delta \gamma<\sqrt{2\left(\rho^{2} \gamma^{2}-\sigma_{\gamma}^{2}\right)}
$$

with the preferred value obviously towards the bottom end of this range. Interestingly, for NGLS parameters and $\rho=7.8 \times 10^{-4}$ at resonant wavelength of $1 \mathrm{~nm}$ (as determined from the Xie formulae) a value of $\Delta \gamma$ that satisfies criterion (3) can be found for all $n \leq 26$, whereas if the natural energy spread was doubled to $200 \mathrm{keV}$ then (3) is only satisfied for all $n \leq 13$. This illustrates that the success of the FEL schemes presented in this report depends strongly on the beam energy spread being small enough.

With the modulator lengths set to give the appropriate energy modulation, the chicane strengths are adjusted to maximise the bunching factor at the required harmonic at the entrance to the next undulator section, whether that be a modulator or a final radiator.

This approach was adopted to set up three alternative configurations, in each case only considering output at $1 \mathrm{~nm}$ from the final radiator. The first was a scheme with a single harmonic conversion from $12 \mathrm{~nm}$ to the 12 th harmonic at $1 \mathrm{~nm}$, as shown schematically in Figure 5, the second was a scheme with one harmonic conversion to the 3rd harmonic at $4 \mathrm{~nm}$ then a subsequent harmonic conversion to the 4 th harmonic at $1 \mathrm{~nm}$, as shown in Figure 6, then finally the same scheme with two harmonic conversions, but using a reduced seed power of $40 \mathrm{~kW}$. In each case the same optimisation procedure was used to allow a fair performance comparison. The results of time-dependent simulations of the three schemes are shown in Sections 3.1, 3.2 and 3.3.

\section{Time Dependent Simulations}

The time-dependent simulations were done using Genesis 1.3. At the time this work was done a full simulated bunch distribution was not available, so full start-to-end modelling could not be done. Instead an ideal rectangular electron bunch of peak current $I_{\text {peak }}=1000 \mathrm{~A}$ was used, with the slice emittance and energy spread also constant along 

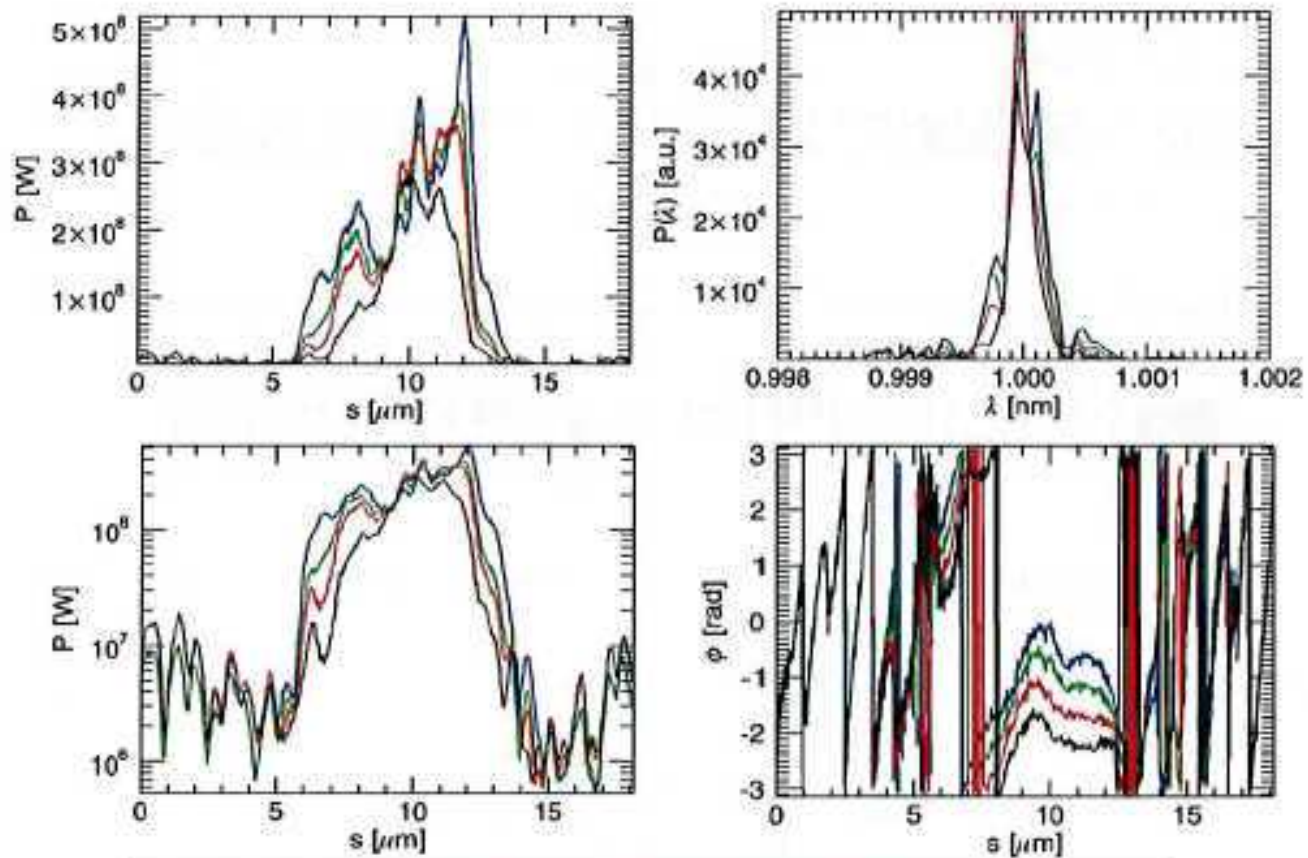

$$
\text { KEY: } \quad B=0.175 T \quad B=0.165 T \quad B=0.155 T \quad B=0.145 T
$$

Figure 7: Single harmonic conversion scheme: Genesis 1.3 time-dependent simulation results. Shown are the radiation power on linear scale (top left), the radiation spectrum (top right), the radiation lower on log scale (bottom left) and the radiation phase (bottom right), all at a propagation distance through the radiator undulator of $50 \mathrm{~m}$.

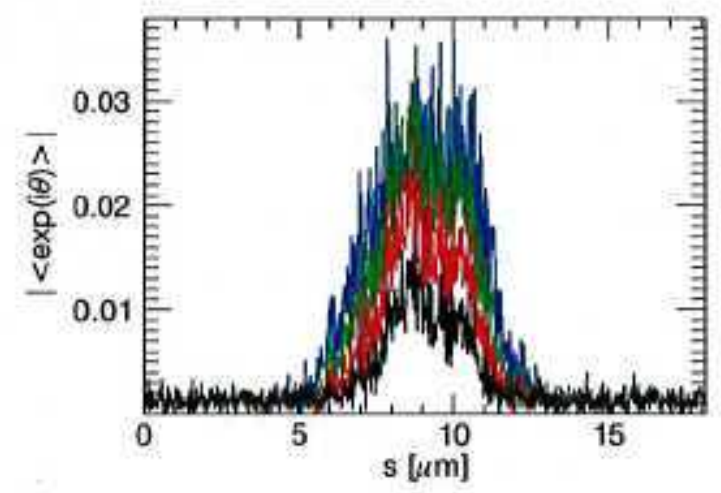

Figure 8: Single harmonic conversion scheme: bunching at entrance to radiator. As in Figure 7, the different colours correspond to varying strengths of the chicane prior to the radiator.

the bunch with values as given in Table 1 . The seed pulse was a perfect gaussian of constant phase. 


\subsection{Single Harmonic Conversion}

Figure 7 shows the radiation power on linear scale (top left), the radiation spectrum (top right), the radiation lower on $\log$ scale (bottom left) and the radiation phase (bottom right), all at the saturation length $L_{\mathrm{sat}}=50 \mathrm{~m}$ through the radiator undulator, with saturation being determined as the point where the bandwidth of the radiation pulse is minimum. Four overlaid pulses are shown, each corresponding to a different field strength of the chicane prior to the radiator. The chicane is modelled in Genesis 1.3 as four dipoles, each of field strength $B$ and length $L_{D}=0.124 \mathrm{~m}$, separated by drift spaces of $0.124 \mathrm{~m}$. Of the four pulses the optimum is taken to be that corresponding to $B=0.145 \mathrm{~T}$ as this pulse has the smoothest temporal profile. The saturation power is therefore $P_{\mathrm{sat}} \simeq$ $300 \mathrm{MW}$, with a contrast ratio of $R \simeq 60$. Further analysis of the output pulse, including pulse length, bandwidth and time-bandwidth product, is given in Table 2 to allow direct comparison with the results from the alternative FEL configurations studied. Figure 8 shows the bunching factor $b_{0}$ at the entrance to the radiator - for the 'optimum' case of $B=0.145 \mathrm{~T} b_{0}$ peaks at 0.015 . 


\subsection{Two Harmonic Conversions}
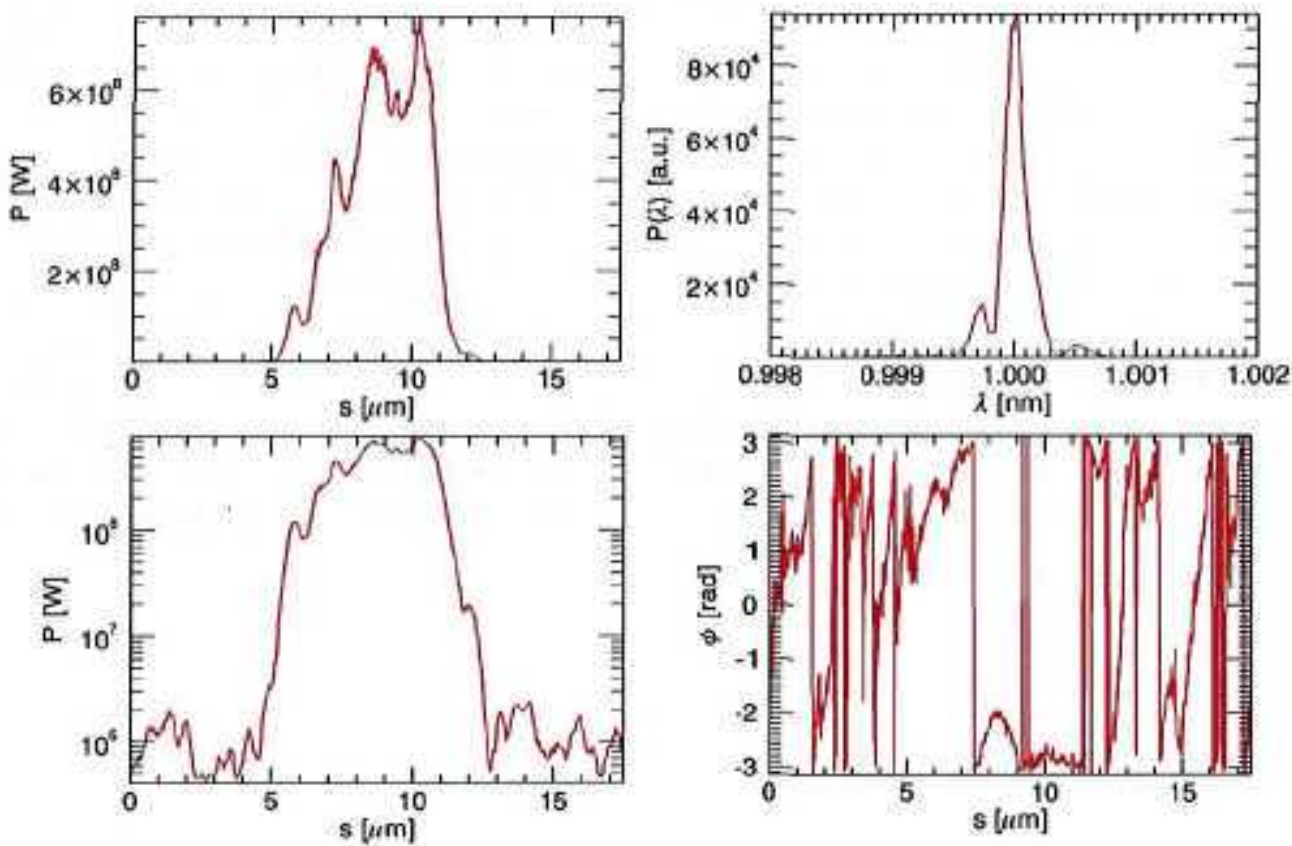

Figure 9: Double harmonic conversion scheme: Genesis 1.3 time-dependent simulation results. Shown are the radiation power on linear scale (top left), the radiation spectrum (top right), the radiation lower on log scale (bottom left) and the radiation phase (bottom right), all at a propagation distance through the radiator undulator of $40 \mathrm{~m}$

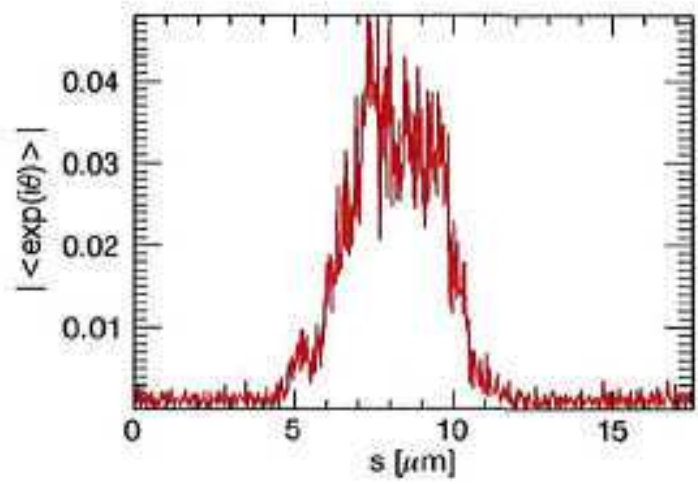

Figure 10: Double harmonic conversion scheme; bunching at entrance to radiator.

The results are shown in Figure 9 for the optimum chicane field strength only. This configuration demonstrates superior performance, with peak power increased to $P_{\text {sat }} \simeq$ $300 \mathrm{MW}$ at a reduced saturation length of $L_{\mathrm{sat}}=50 \mathrm{~m}$. The contrast ratio is increased by an order of magnitude to $R \simeq 700$. The improved performance is due to stronger bunching at the start of the radiator, as illustrated in Figure 10 . Now $b_{0}$ peaks at nearly 0.05 so 


\begin{tabular}{llll}
\hline & $\begin{array}{l}\text { Single } \\
\text { Conversion }\end{array}$ & $\begin{array}{l}\text { Two } \\
\text { Conversions }\end{array}$ & $\begin{array}{l}\text { Reduced } \\
\text { Seed Power }\end{array}$ \\
\hline Saturation Power $P_{\text {sat }}$ & $300 \mathrm{MW}$ & $750 \mathrm{MW}$ & $1.06 \mathrm{GW}$ \\
Contrast Ratio $R$ & 60 & 700 & 350 \\
FWHM Pulse duration $\Delta t$ & $9.5 \mathrm{fs}$ & $13 \mathrm{fs}$ & $11 \mathrm{fs}$ \\
FWHM Bandwidth $\Delta \lambda / \lambda$ & $1.9 \times 10^{-4}$ & $1.8 \times 10^{-4}$ & $2.5 \times 10^{-4}$ \\
Time-Bandwidth product $\Delta \nu \Delta t$ & 0.54 & 0.73 & 0.84 \\
Radiator Saturation Length $L_{\text {sat }}$ & $50 \mathrm{~m}$ & $40 \mathrm{~m}$ & $40 \mathrm{~m}$ \\
Modulator/chicanes length & $12 \mathrm{~m}$ & $12 \mathrm{~m}$ & $16 \mathrm{~m}$ \\
Total System Length & $62 \mathrm{~m}$ & $52 \mathrm{~m}$ & $56 \mathrm{~m}$ \\
\hline
\end{tabular}

Table 2: Summary of three alternative configurations.

the intitial burst of colerent emission over the first few gain lengths in the radiator is stronger, scaling as $P_{\text {coh }} \propto b_{0}^{2}$.

\subsection{Reduced Seed Power}

Finally shown, in Figures 11 and 12 are the results of the case reoptimised for a reduced seed power of $P_{\text {seod }}=40 \mathrm{~kW}$. The output pulse quality is clearly degraded here, both temporally and spectrally, but still temporally coherent as demonstrated by the slow variation of radiation phase along the pulse. Such a pulse would undoubtably be suitable for some applications where a SASE pulse would be unusable.

\subsection{Comparison Summary}

The comparative performance of the three alternative configurations, each set up to produce $1 \mathrm{~nm}$ FEL output, is summarised in Table 2. The Total System Length is from the start of the first modulator undulator to the saturation point within the radiator. Each scheme can produce temporally coherent FEL pulses, with peak power of several hundred megawatt, within the space constraint imposed of $\simeq 60 \mathrm{~m}$. Superior performance is obtained from the scheme in which two harmonic conversions are used to convert the coherent HHG seed pulse at $12 \mathrm{~nm}$ to coherent FEL output at $1 \mathrm{~nm}$. Acceptable performance may be achievable with further reductions in seed power below $40 \mathrm{~kW}$, possibly in combination with the use of optical klystron modulator undulators. 

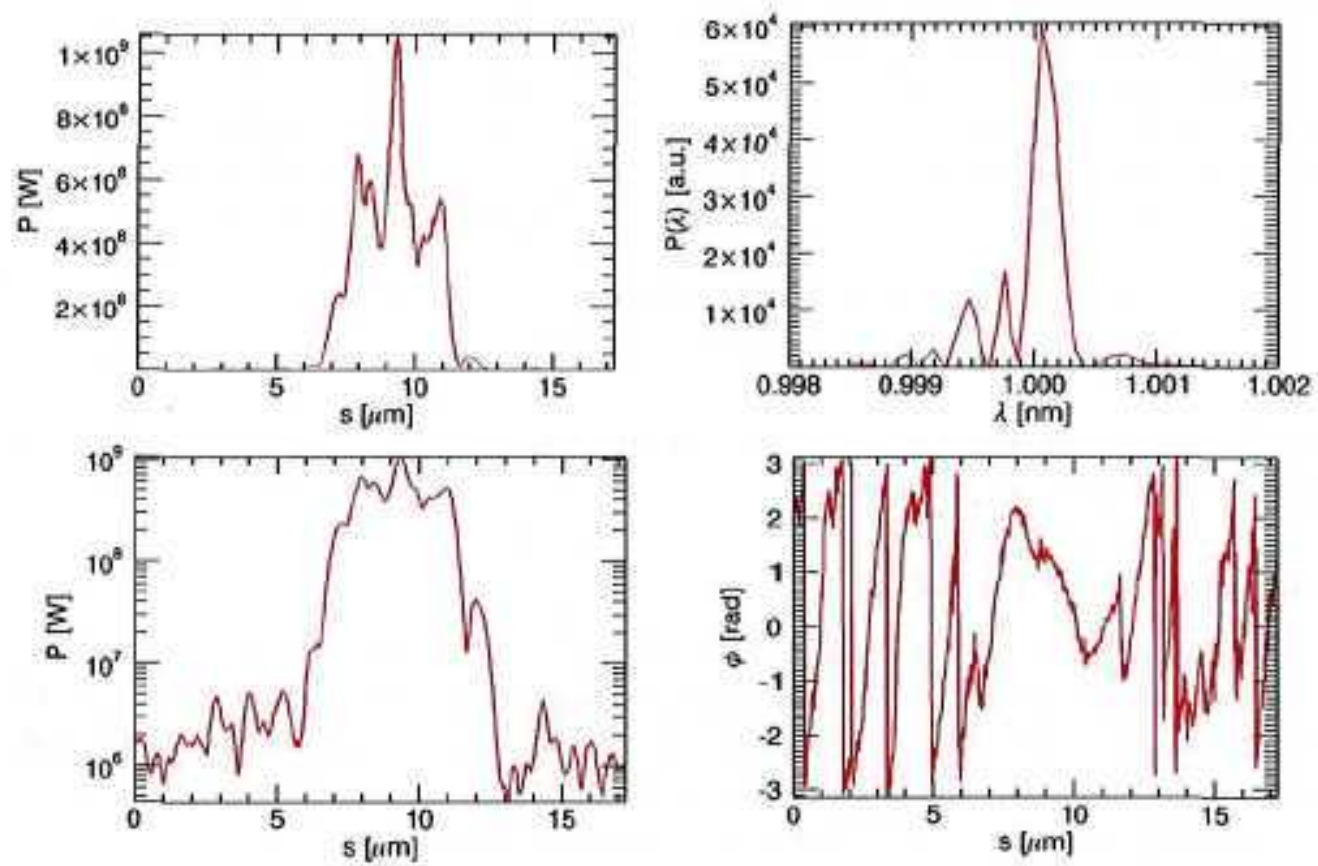

Figure 11: Reduced Seed Power: Genesis time-dependent simulation results. Shown are the radiation power on linear scale (top left), the radiation spectrum (top right), the radiation lower on log scale (bottom left) and the radiation phase (bottom right), all at a propagation distance through the radiator undulator of $40 \mathrm{~m}$

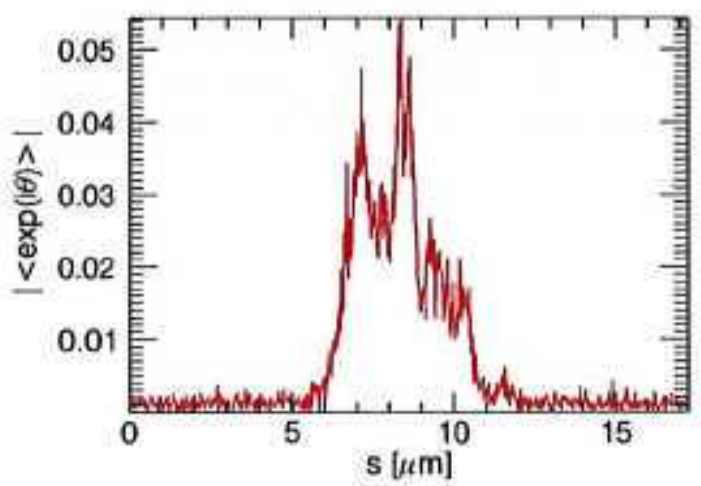

Figure 12: Reduced Seed Power: bunching at entrance to radiator. 


\section{Harmonic Afterburners}

The schemes proposed in the previous sections can be extended to produce coherent radiation pulses at higher harmonics of the radiator resonant wavelength. This can be done by exploiting the harmonic bunching in the electron beam which is provided 'for free' by the FEL interaction at the fundamental resonant wavelength. A short undulator module, called a 'harmonic afterburner', can be placed after the radiator, tuned to a higher harmonic. Because the beam is strongly pre-bunched at the resonant wavelength of this afterburner there is a strong burst of coherent emission which grows quadratically in power with propagation distance over the first couple of gain lengths. The energy spread already induced by the FEL interaction at the fundamental prohibits this harmonic radiation entering the exponential growth regime, with power growing only linearly after the initial colerent emission. Nervertheless, the power emitted in this initial quadratic burst can be substantial. In theory, the afterburner axis could be at a slight angle from the FEL radiator axis, with a weak deffection of the electron beam onto the afterburner axis. The temporally synchronised fundamental and harmonic emission will then become spatially separated some distance downstream. Of course the beam deflection must be small enough to preserve the harmonic bunching in the electron beam (or an isochronous bend must be designed). The maximum angle can therefore only be defined after further analysis.

\subsection{Simulations}

Simulations have been done based on the NGLS scheme that uses two harmonic conversions (described in Section 3.2). The parameters chosen for the afterburners are planar geometry and periods $\lambda_{w}=14.5 \mathrm{~mm}$ for resonance at $2 \mathrm{nd}$ harmonic $(0.5 \mathrm{~nm})$ and $\lambda_{w}=12.0 \mathrm{~mm}$ for resonance at third harmonic. The undulators are set to minimum gap of $5 \mathrm{~mm}$ giving $\bar{a}_{w} \simeq 0.7$ at $0.5 \mathrm{~nm}$ and $\bar{a}_{w} \simeq 0.5$ at $0.33 \mathrm{~nm}$. Gap tuning to longer wavelengths is thus not possible this is a consequence of the fact that the electron beam energy is appropriate for good FEL coupling and reasonable gap tuning at $1 \mathrm{~nm}$ resonance to obtain the same tunability at shorter wavelengths would require a higher beam energy.

The afterburner undulator is placed immediately after the saturation point of the $1 \mathrm{~nm}$ radiation in the radiator. No attempt has been made to adjust the position to maximise the bunching at the required harmonic. At this point the bunching parameters for the 2nd and 3rd harmonics, averaged over the short section of the electron beam that has interacted with the initial seed, are $b_{2} \simeq 0.08$ and $b_{3} \simeq 0.04$, as shown in the top left panels of Figures 14 and 15 respectively. Also shown in Figures 14 and 15 for $0.5 \mathrm{~mm}$ and $0.33 \mathrm{~nm}$ respectively are: the radiation power growth through the undulators (top right); the pulse profiles after $4 \mathrm{~m}$ propagation through the undulator (bottom left); the radiation spectrums, also at $4 \mathrm{~m}$ propagation through the undulator (bottom right).

The results show that after only $4 \mathrm{~m}$ of undulator $64 \mathrm{MW}$ of coherent power can be extracted at the $2 \mathrm{nd}$ harmonic of $1 \mathrm{~nm}$, and nearly $20 \mathrm{MW}$ at the $3 \mathrm{rd}$ harmonic, with a contrast ratio of $R>200$ in each case. The temporal coherence of the $0.5 \mathrm{~nm}$ pulse is good with a calculated time-bandwidth product of $\Delta \nu=0.81$, approximately twice that

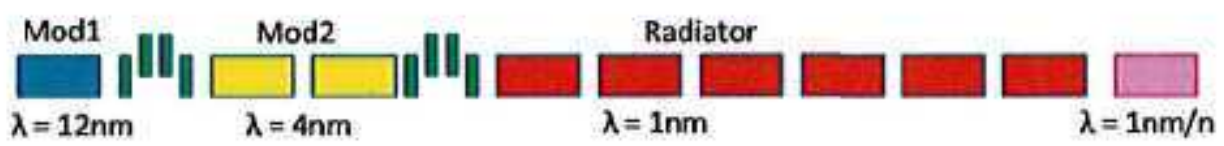

Figure 13: Schematic of the use of a harmonic afterburner (shown in pink). 


\begin{tabular}{lll}
\hline & 2nd Harmonic & 3rd Harmonic \\
\hline Wavelength & $0.5 \mathrm{~nm}$ & $0.33 \mathrm{~nm}$ \\
Peak Power (at $z=4 \mathrm{~m}$ ) $P_{\text {peak }}$ & $64 \mathrm{MW}$ & $19 \mathrm{MW}$ \\
Contrast Ratio $R$ & 220 & 300 \\
FWHM Pulse duration $\Delta t$ & $13 \mathrm{fs}$ & $20 \mathrm{fs}$ \\
FWHM Bandwidth $\Delta \lambda / \lambda$ & $5.0 \times 10^{-5}$ & - \\
Time-Bandwidth product $\Delta \nu \Delta t$ & 0.81 & - \\
\hline
\end{tabular}

Table 3: Output summary for 2nd and 3rd harmonic afterburners.

of a transform limited gaussian pulse. The calculated spectrum for the $0.33 \mathrm{~mm}$ case is very noisy, prohibiting a meaningful calculation of the time-bandwidth product. Care was taken that the number of macroparticles per slice used in the simulations, as well as the parameters used in the code to construct the initial bunch distribution, were appropriate for the harmonic ratio between the initial seed and final output at the afterburner resonant wavelength. However, more work is required to assess whether or not the spectral noise at $0.33 \mathrm{~nm}$ is a numerical artefact.

\section{References}

[1] New Light Source Project: Conceptual Design Report, 2010.

[2] C. J. Bochetta. Fermi@elettra Conceptual Design Report, 2007.

[3] Ming Xie. Design Optimization for an X-Ray Free Electron Laser Driven by SLAC Linac. In 1995 Particle Accelerator Conference, pages 183-185, 1995. 

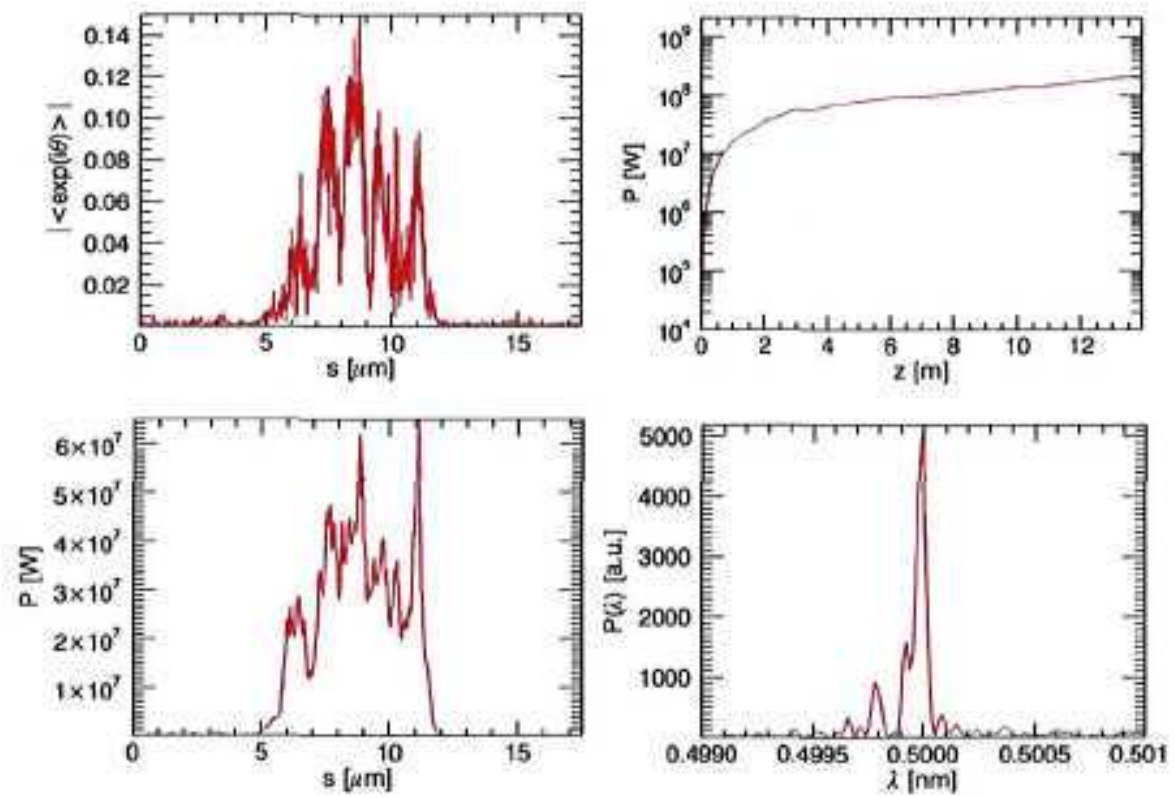

Figure 14: 2nd Harmonic Afterburner: Bunching parameter at entrance to afterburner undulator (top left); the radiation power growth through the undulator (top right); the pulse profile after $4 \mathrm{~m}$ propagation through the undulator (bottom left); the radiation spectrum, also at $4 \mathrm{~m}$ propagation through the undulator (bottom right).
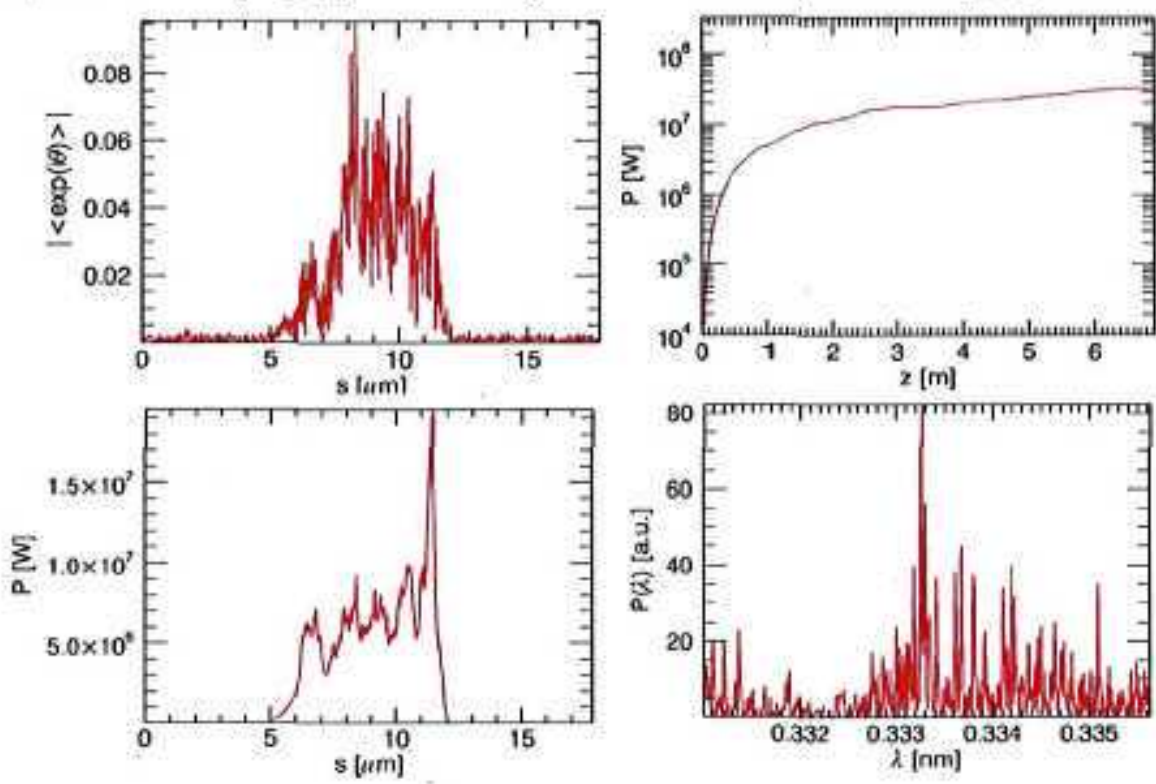

Figure 15: 3rd Harmonic Afterburner: Bunching parameter at entrance to afterburner undulator (top left); the radiation power growth through the undulator (top right); the pulse profile after $4 \mathrm{~m}$ propagation through the undulator (bottom lelt); the radiation spectrum, also at $4 \mathrm{~m}$ propagation through the undulator (bottom right). 


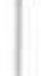

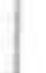

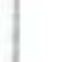

